\title{
Original
}

\section{An Analysis of Factors Associated with Airway Hyperresponsiveness in Mild to Moderate Asthma}

\author{
Kumiko Shiba, Keita Kasahara, Tetsuji Ozawa \\ and Mitsuru AdachI
}

\begin{abstract}
The aim of this study was to examine the association between airway hyperresponsiveness (AHR) in asthmatic patients and various factors, including the duration of asthma, the thickness of the epithelial reticular basement membrane $(\mathrm{Rbm})$, and eosinophilic inflammation or total IgE in blood. We measured the thickness of $\mathrm{Rbm}$ in bronchial biopsy specimens. Spirometric tests and histamine challenge tests were also performed to evaluate airflow obstruction and AHR. Multiple regression analysis was used to assess the correlation between PC20 histamine values with duration of asthma, thickness of Rbm, eosinophil percentage in sputum and blood, and total IgE in blood. Furthermore, we used stepwise regression analysis to determine which factors were closely correlated with AHR. This study showed that AHR was most associated with the thickness of Rbm. The results suggest that the prevention and improvement of the airway structural changes should be considered as one of the targets of asthma treatment.
\end{abstract}

Key words : asthma, airway hyperresponsiveness, airway structural change, epithelial reticular basement membrane, steroid inhalation

\section{Introduction}

One of the characteristics of bronchial asthma is airway hyperresponsiveness (AHR $)^{1-4)}$, therefore effective inhibition of AHR would be very useful in the treatment of asthma. Various factors have been reported to be associated with AHR : eosinophilic inflammation; structural change of airways; duration of asthma; and IgE concentration.

High levels of eosinophils in sputum and blood have been detected in asthmatic patients, even in those not having attacks ${ }^{5)}$. Furthermore, thickening of the epithelial reticular basement membrane $(\mathbf{R b m})$ of the airway wall was also found even in patients with mild asthma $^{6,7)}$. Some studies have suggested that the inflammation and detailed background mechanisms which induce structural changes of the airway wall might also induce $\mathrm{AHR}^{8-10)}$. In addition, other reports have indicated that deterioration of respiratory function is influenced by the duration of asthma and the $\operatorname{IgE}$ concentration $^{11)}$. It has recently been suggested that anti-inflammatory treatment is the most important treatment for asthma $^{11,12)}$. However, some investigators have reported that airway hyprerresponsiveness could not be reduced despite intensive treatment with steroids ${ }^{7,13-15)}$. Therefore, it is not clear which of these factors are strongly contributing to AHR in patients with asthma. 
Clinically, we are not sure which specific therapeutic target we should aim for in the treatment of asthma: inhibition of airway inflammation; prevention or improvement of airway remodeling; reduction of IgE concentration; or other targets. We would like to clearly identify the factors which are closely associated with AHR so that therapy can be more accurately prescribed.

\section{Materials and Methods}

\section{Subjects}

We examined 32 patients (age range, 20 to 67) with mild to moderate asthma (Table 1). Ten patients had been treated with inhaled corticosteroids $(200$ to $800 \mathrm{~g} /$ day beclomethasone) and 22 patients used only bronchodilators to alleviate their asthmatic symptoms. Asthma was diagnosed and classified according to Guidelines for the Diagnosis and Management of the Second Expert Panel Report, 1997 ${ }^{16)}$ with reversibility of forced expiratory volume in one second $($ FEV1) in response to bronchodilation of $>15 \%$. All subjects were non-smokers. No subjects were suffering from chronic obstructive pulmonary disease (COPD), bronchiectasis or inactive pulmonary tuberculosis, which was confirmed by symptom checks, physical examinations, chest roentgenographic and computed tomography (CT) examinations. All subjects were informed of the details and their consents were obtained prior to the commencement of this study.

\section{Study design}

Patients were screened by history, physical examination and respiratory function. After ascertaining that their asthma conditions were stable and their medications had remained unchanged for at least three months, a standard histamine challenge test and bronchoscopy were performed. First, we evaluated the relationship between hyperresponsiveness (PC20 histamine) and other factors (duration of asthma, thickness of the epithelial reticular basement membrane ( $\mathrm{Rbm})$, eosinophil level in induced sputum (\%spEo) and in blood (\%seEo), and total IgE by multiple regression and stepwise analysis.

Table 1. Subject characteristics

\begin{tabular}{ll}
\hline & 32 asthmatics \\
\hline Age $(\mathrm{yrs})$ & $37.1(12.5)$ \\
Sex M : F & $18: 14$ \\
Atopy : Non-atopy & $14: 18$ \\
BDP $(+):(-)$ & $10: 22$ \\
$\%$ FEV $(\%)$ & $77.4(14.1)$ \\
PC $_{20}$ histamine $(\mu \mathrm{g} / \mathrm{ml})$ & $1881(2833)$ \\
\hline
\end{tabular}

Definition of abbreviations :

$\mathrm{BDP}=$ beclometasone dipropionate

$\mathrm{PC}_{20}$ histamine $=$ concentration of histamine causing $20 \%$ decrease in FEV,

FEV , forced expiratory volume in one second

$\% \mathrm{FEV}_{1}=\mathrm{FEV}_{1} /$ predicted $\times 100$

Values are expressed as mean (SD). 
Respiratory function tests

Spirometric tests were performed with a spirometer (Autospiro AS-300, Minato Co., Osaka, Japan) to measure forced vital capacity (FVC) and forced expiratory volume in one second (FEV1). The tests were repeated at least three times and the highest acceptable measurement was used for analysis.

\section{Histamine challenges}

Bronchial responsiveness to histamine was measured by a method described by guidelines produced by the Japanese Society of Allergology ${ }^{17)}$. Subjects inhaled doubling concentrations of histamine by De Villbis nebulizer for two minutes by tidal breathing. Increasing concentrations of histamine were administered until FEV1 decreased by $>20 \%$ of the baseline value. Results were expressed as the provocative concentration that caused a $20 \%$ decrease in FEV1 from the post-saline baseline (PC20 histamine). PC20 histamine values were log-transformed before analysis.

\section{Bronchial biopsy}

Bronchial biopsy was performed in all subjects using a bronchofiberscope examination (BF type240, Olympus Co., Tokyo, Japan). Before bronchofiberscopic examination, we ensured an absence of asthma attacks in the subjects. We used lidocaine $(20 \mathrm{ml}$ of $2 \%$ solution for local anesthesia), atropine $(0.5 \mathrm{mg}$ intramuscularly) and midazolam $(0.1 \mathrm{mg} / \mathrm{kg}$ by slow intravascular infusion) as premedications. We started bronchofiberscopy by monitoring the percutaneous oxygen saturation and pulse by pulse oximeter (Pulsox, Teijin Co., Tokyo, Japan), as well as respiratory rate and blood pressure. Four to five biopsy specimens were taken from the subsegmental bronchial bifurcation in the right lower lobe in each subject. The outer sites of biopsy specimens were stained by eosin to ensure proper orientation for cross-sectioning. After fixation in $10 \%$ buffered formalin solution at room temperature, the specimens were embedded in paraffin, cut into five micrometer sections with a microtome, and stained with hematoxylin-eosin. Three measurable stained sections were obtained per subject. Rbm thickness was quantified by measuring the area of the whole Rbm (from the base of the bronchial epithelium to the outer limit of the reticular lamia) and the length of the true basement membrane of the Rbm area using a computerized image (Apple Computer, Cupertino, CA, NIH image). Rbm thickness was calculated by the $\mathrm{Rbm}$ area/the length of the basement membrane. We used the average value from three to five measurements in three to five stained sections per subject for analysis. A single pathologist examined all sections blindly.

\section{Sputum induction and processing}

Sputum induction was performed as described previously ${ }^{18)}$. Briefly, sputum was induced by inhalation of increasing concentrations of hypertonic saline $(0.9,1.8,3.0,4.0$, and 5.0\%) until an adequate sample was collected. Subjects were instructed to cough deeply in five-minute intervals. Gargling before and between each induced cough was encouraged to minimize salivary contamination. In sputum, cell plugs were separated from saliva and treated with Wright-Giemsa stain so that inflammatory cells could be counted. The percentage of inflammatory cells was determined by counting 900 cells under a light microscope. 
Table 2. Factors for airway hyperresponsiveness - multiple regression analysis-

\begin{tabular}{lcccccc}
\hline & mean (SD) & $\begin{array}{c}\text { ragression } \\
\text { coefficient }\end{array}$ & 95\%low & 95\%high & p value & F value \\
\hline duration (years) & $8.5(12.5)$ & -0.008 & -0.028 & 0.011 & 0.39 & 0.78 \\
Rbm thickness ( $\mu$ m) & $7.1(2.3)$ & -0.149 & -0.281 & -0.016 & 0.03 & 5.33 \\
\%spEo (\%) & $23.9(22.6)$ & -0.008 & -0.021 & 0.004 & 0.19 & 1.85 \\
\%seEo (\%) & $6.8(7.7)$ & 0.017 & -0.018 & 0.052 & 0.33 & 0.97 \\
IgE in serum (IU) & $558.6(790.8)$ & 0.00017 & -0.00016 & 0.0005 & 0.31 & 1.10 \\
\hline
\end{tabular}

Definition of abbreviations :

$\mathbf{R b m}=$ epithelial reticular basement membrane

$\% \mathrm{spEo}=$ percentage of eosinophils in induced sputum

$\%$ seEo $=$ percentage of eosinophils in biood

Values are expressed as mean (SD)

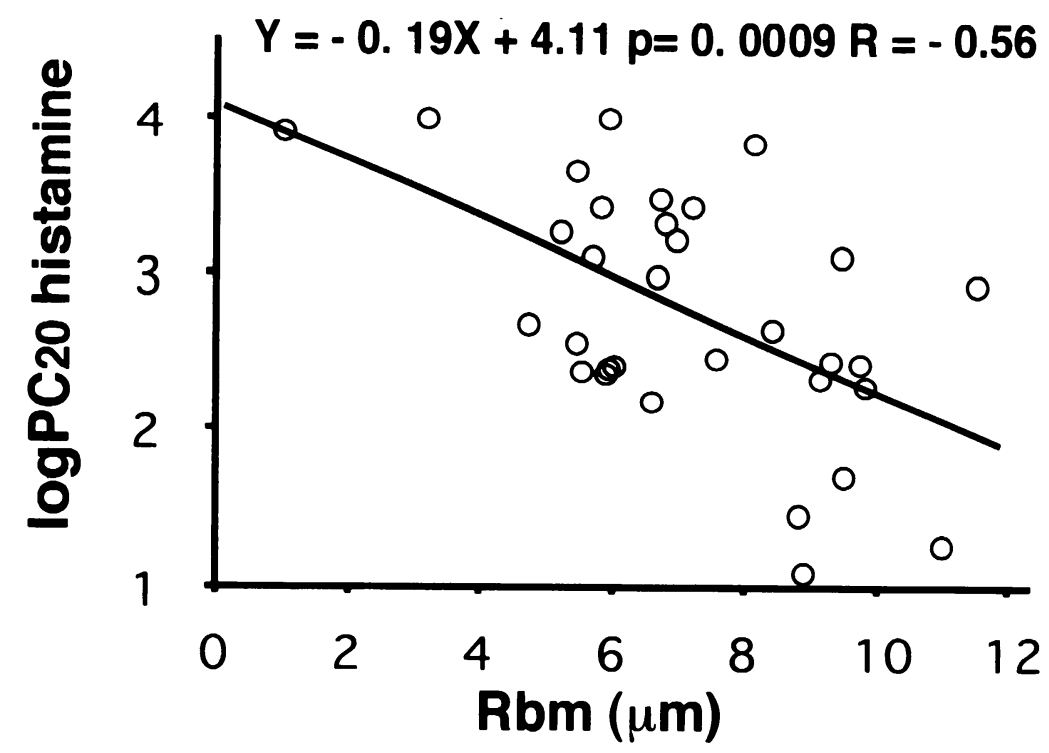

Fig. 1. Relationships between bronchial Rbm thickness and $\log P C 20$ histamine in patients with mild to moderate asthma.

The logPC20 histamine was negatively correlated with Rbm thickness $(p=0.0009 R=-0.56)$

\section{Statistical analysis}

All statistical analyses were done using Stat View software version 4.5 (Abacus Concepts, Berkeley, CA) on a Macintosh computer. Values were expressed as the mean \pm standard deviation (SD). Multiple regression analysis was performed to assess the contributions of duration of asthma, thickness of Rbm, percentage of eosinophils in sputum or blood, and total IgE to AHR ( $\log$ PC20 histamine). Differences with $\mathrm{p}$ values less than 0.05 were considered significant. 


\section{Results}

\section{Subject characteristics}

The characteristics of the subjects are presented in Table 1 including: age; sex ; presence of atopy; use of beclomethasone dipropionate (BDP) ; \%FEV1; and PC20 histamine value in 32 patients with asthma. Bronchial biopsy was performed in all subjects. PC20 histamine values ranged from 12 to $10,000 \mu \mathrm{g} / \mathrm{ml}($ mean $\pm \mathrm{SD}: 1881 \pm 2833 \mu \mathrm{g} / \mathrm{ml}$ ).

Correlation between PC20 histamine and duration of asthma, thickness of Rbm, \%spEo, $\%$ seEo, and $\operatorname{IgE}$

We examined the correlations between PC20 histamine and five factors (duration of asthma, thickness of $\mathrm{Rbm}$, \%spEo, \% seEo, and $\mathrm{IgE}$ ) using multiple regression analysis. The value of PC20 histamine was significantly correlated with the thickness of Rbm $(\mathrm{F}=5.33, \mathrm{p}=0.03)$. However, the other factors showed no significant correlation (Table 2). We adopted only the thickness of $\mathrm{Rbm}$ for the linear formula by stepwise regression analysis $\left(\log\right.$ PC20 histamine $=4.11-0.19^{*}$ thickness of $\left.\mathrm{Rbm}: \mathrm{p}=0.0009 \mathrm{R}=-0.56\right)$ (Fig. 1$)$.

\section{Discussion}

In this study, we analyzed 32 mild or moderate asthmatic patients by a stepwise regression method to clarify which factors are correlated most closely with AHR. We examined the duration of asthma, the thickness of Rbm, eosinophil percentage in sputum, eosinophil percentage in peripheral blood and IgE in serum. These results showed that the thickness of Rbm most affected PC20 histamine, which was the index for AHR, considering the regression coefficient, $p$ value and $F$ value of the multiple regression analysis. These results also suggested that the thickening of $\mathrm{Rbm}$ might be strongly associated with AHR. Previous studies also indicated that the structural changes of the bronchial wall might induce airflow limitation and AHR in asthma ${ }^{9,10)}$. Furthermore, thickening of the Rbm was reported to parallel the thickness of the whole airway wall ${ }^{19)}$. The findings of the present and previous studies suggest that the airway structural changes could play a very important role in the induction of AHR and airflow limitation.

Several previous reports have suggested that airway eosinophilic inflammation is an important factor in deterioration of respiratory functions and development of persistent airway narrowing in patients with asthma ${ }^{20,21)}$. We also speculate that eosinophil inflammation in airways may cause hyperresponsiveness by inducing structural changes. In this study, eosinophil percentage in sputum had the second largest correlation with PC20 histamine after the thickness of bronchial $\mathrm{Rbm}$, however the correlation was not statistically significant. We evaluated the eosinophil percentage in sputum at only one time point. While eosinophilic inflammation induces airway structural changes, these changes may depend on both the intensity and duration of inflammation. This study did not show a statistically significant association between eosinophil percentage in sputum and AHR. We are also aware that some examinations have suggested that AHR did not improve with steroid therapy while eosinophilic inflammation of airway was decreasing ${ }^{11,22-24)}$. To obtain conclusive evidence, we would need new strictly designed studies to examine whether eosinophilic inflammation can be associated with AHR.

In this study we suggested that the thickening of bronchial Rbm might be the factor 
most closely associated with AHR. The structural change of airway walls could be associated with the deterioration of respiratory function of asthma through AHR. The results suggest that the prevention and improvement of the airway structural changes should be considered as an important target for asthma treatment.

\section{References}

1) Poston RN, Chanez P, Lacoste JY, Litchfield T, Lee TH and Bousquet J: Immunohistochemical characterization of the cellular infiltration in asthmatic bronchi. Am Rev Respir Dis $145: 918-921$ (1992)

2) O'Byrine PM, Hargreeve FE and Kirby JG : Airway inflammation and hyperresponsiveness. Am Rev Respir Dis 136(4 Pt 2) : S35-S37 (1987)

3) Boulet LP, Turcotte H, Laviolette M, Naud F, Bernier MC, Martel S and Chakir J: Airway hyperresponsiveness, inflammation, and subepithelial collagen deposition in recently diagnosed versus long-standing mild asthma. Influence of inhaled corticosteroids. Am J Respir Crit Care Med 162:1308-1313 (2000)

4) Chetta A, Foresi A, Del Donno M, Consigli GF, Bertorelli G, Pesci A, Barbee RA and Olivieri D : Bronchial responsiveness to distilled water and methacholine and its relationship to inflammation and remodeling of the airways in asthma. Am J Respir Crit Care Med 153 : 910-917 (1996)

5) Walker C, Bauer W, Braun RK, Menz G, Braun P, Schwarz F, Hansel TT and Villiger B: Activated T cells and cytokines in bronchoalveolar lavages from patients with various lung diseases associated with eosinophilia. Am J Respir Crit Care Med 150 : 1038-1048 (1994)

6) Boulet LP, Chakir J, Dube' J, Laprise C, Boulet M and Laviolette M: Airway inflammation and structural changes in airway hyperresponsiveness and asthma: an overview. Can Respir J 5:16-21 (1998)

7) Laprise C, Laviolette $\mathbf{M}$, Boulet $\mathbf{M}$ and Boulet LP: Asymptomatic airway hyperresponsiveness: relationships with airway inflammation and remodeling. Eur Respir J 14:63-73 (1999)

8) Hoshino M, Nakamura $Y$ and Sim JJ : Expression of growth factors and remodeling of the airway wall in bronchial asthma. Thorax $53: 21-27$ (1998)

9) Chetta A, Foresi A, Del Donno M, Bertorelli G, Pesci A and Olivieri D: Airways remodeling is a distinctive feature of asthma and is related to severity of disease. Chest $111: 852-857$ (1997)

10) Boulet LP, Laviolette M, Turcotte H, Cartier A, Dugas M, Malo JL and Boutet M: Bronchial subepithelial fibrosis correlates with airway responsiveness to methacholine. Chest 112:45-52 (1997)

11) Selroos $\mathrm{O}$, Pietinalho $\mathrm{A}$, Lofroos $\mathrm{AB}$ and Riska $\mathrm{H}$ : Effect of early vs late intervention with inhaled corticosteroids in asthma. Chest $108: 1228-1234$ (1995)

12) Donahue JG, Weiss ST, Livingston JM, Goetsch MA, Greineder DK and Platt R: Inhaled steroids and the risk of hospitalization for asthma. JAMA $227: 887-891$ (1997)

13) Oddera S, Silvestri M, Balbo A, Jovovich BO, Penna R, Crimi E and Rossi GA: Airway eosinophilic inflammation, epithelial damage, and bronchial hyperresposiveness in patients with mild-moderate, stable asthma. Allergy 51 : 100-107 (1996)

14) Djukanovic R, Wilson JW, Britten KM, Wilson SJ, Walls AF, Roche WR, Howarth PH and Holgate ST: Effect of inhaled corticosteroid on airway inflammation and symptoms in asthma. Am Rev Respir Dis 145:669674 (1992)

15) Vathenen AS, Knox AJ, Wisniewski A and Tattersfield AE: Time course of change in bronchial reactivity with an inhaled corticosteroid in asthma. Am Rev Respir Dis 143 : 1317-1321 (1991)

16) Second Expert Panel Report Guidelines for the diagnosis and management of asthma. NIH Publication 15-24 (1997)

17) Makino S, Kobayashi S, Miyamoto T, Shida T and Takahashi S: Standard for allergen bronchial provocation test asthma and hypersensitivity pneumonitis. Jpn J Allergy 31 : 1074-1076 (1982) (in Japanese)

18) Minoguchi K, Kohno Y, Oda N, Wada K, Miyamoto M, Yokoe T, Hashimoto T, Akabane T, Kobayashi H, Mita S, Kihara $\mathbf{N}$ and Adachi $\mathbf{M}$ : Effect of theophylline withdrawal on airway inflammation in asthma. Clin Exp Allergy 28(Suppl 3) : 57-63 (1998)

19) Kasahara K, Shiba K, Ozawa T, Okuda $K$ and Adachi $M$ : Correlation between the bronchial subepithelial layer and whole airway wall thickness in patients with asthma. Thorax 57:242-246 (2002)

20) Crimi E, Spanevello A, Neri M, Ind PW, Rpssi GA and Brusasco V: Dissociation between airway inflammation and airway hyperresponsiveness in allergic asthma. Am J Resir Crit Care Med 157:4-9 (1998)

21) Fish JE and Peters SP: Airway remodeling and persistent airway obstruction in asthma. J Allergy Clin Immunol 104 : 509-516 (1999)

22) Haahtela T, Jarvinen M, Kava T, Kiviranta K, Koskinen S, Lehtonen K, Nikander K, Persson T, Selroos O and Sovijarvi A : Effects of reducing or discontinuing inhaled budesonide in patients with mild asthma. $N$ Engl J Med 331 : 700-705 (1994) 
23) Kerstjens HA, Brand PL, Hughes MD, Robinson NJ, Postma DS, Sluiter HJ, Bleecker ER, Dekhuijzen PN, de Jong PM and Mengelers HJ: A comparison of bronchodialator therapy with or without inhaled corticosteroid therapy for obstructive airways disease. $N$ Engl J Med 331:1413-1419 (1992)

24) Overbeek SE, Kerstjens HA, Bogaard JM, Mulder PG and Postma DS : Is delayed introduction of inhaled corticosteroids harmful in patients with obstructive airways disease (asthma and COPD?) The Dutch CNSLD Study Group. The Dutch Chronic Nonspecific Lung Disease Study Groups. Chest 110:35-41 (1996)

[Received January 18, 2002 : Accepted February 20, 2002] 